\title{
Fuzzy Controller for Mechanical Systems
}

\author{
Piotr Kulczycki, Member, IEEE
}

\begin{abstract}
In many applications of motion control, the occurrence of nonlinear friction constitutes a fundamental obstacle in the design of satisfactory controlling systems. Since it is seldom possible to obtain a relatively accurate model of resistance to motion, a solution more and more often applied in practice is to introduce approaches that incorporate the inevitable imprecisions of the model in the form of uncertainties. This paper deals with the time-optimal (minimum-time) control for mechanical systems with a discontinuous and uncertain model of resistance to motion. A fuzzy approach is used in the design of suboptimal feedback controllers, convenient in practice thanks to their many advantages, especially in respect to robustness.
\end{abstract}

Index Terms-Fuzzy models, robustness, suboptimal controller, time-optimal control.

\section{INTRODUCTION}

$\mathbf{P}$ ROBLEMS of time-optimal (minimum-time) control have been considered since the early introduction of optimization theory in control applications. Basic solutions for mechanical systems with a single degree of freedom are well known and can be found in many textbooks (see, e.g., [1, ch. 7.2] or [2, sec. 8]). However, these solutions rely on an object model, and the performance of the obtained control system seems to be very sensitive to occurring mismatches and uncertainties. Therefore, in recent years, many authors have taken up such problems, as well as the robust control techniques needed to cope with them (see in particular [3], [4], and also [5]-[7]). A major source of uncertainty in models of mechanical systems is friction, especially dry friction and stiction effects, and various models for resistance to motion suitable for control purposes have been investigated (see survey paper [8] or book [9]). However, these models mostly include many parameters whose actual values cannot be determined by proper experiments; moreover, they increase the dimensionality of the object model to such an extent that the synthesis of useful time-optimal controlling structures becomes virtually impossible. In the present paper, that problem has been solved by the introduction of the fuzzy type of uncertainty to the discontinuous model for resistance to motion, which has made it possible to propose new types of control structures that take into account the complex nature of friction phenomena, without the undue complication of a control law. Moreover, such a concept also considers the perturbations and noise naturally occurring in the system.

Thus, the dynamics of single-degree-of-freedom mechanical systems with friction can be described in its fundamental version by the differential equation

$$
\ddot{x}(t)=h(\cdot)+u(t)
$$

Manuscript received November 27, 1999; revised April 3, 2000. The author is with the Department of Control Engineering, Cracow University of Technology, PL-31-155 Cracow, Poland (e-mail: kulczycki@ieee.org). Publisher Item Identifier S 1063-6706(00)06586-3. where $x$ denotes the position of an object and $u$ is a bounded control: either a force or a torque depending on the applications. The core of the above model is the function $h$, which represents the resistance to motion and is most often related to friction phenomena that depend predominantly on velocity but, generally, also on position and time, e.g., if there are spring forces and/or gravitational effects. This function can be described in the following form, inspired by the physical perspective:

$$
h(\cdot)=-w \operatorname{sgn}(\dot{x}(t))
$$

where $w$ is a nonnegative real number. The sgn mapping describes the discontinuous nature-with respect to velocity-of friction phenomena. In real systems, however, the number $w$ cannot be sufficiently specified because it represents an overly dispersed range of physical phenomena. In the present paper, this number will be defined as a fuzzy set. In this situation, such a factor takes into account-as a fuzzy uncertainty-the dependence of resistance to motion on a number of factors, e.g., position, as well as velocity or even temperature and disturbances. By its very nature, the above fuzzy approach offers the possibility to describe a complex reality with a precision that exceeds classical modeling techniques. Allowing for some discomfort resulting from the uncertainty introduced into the model (note, e.g., that the state of the dynamical system starts to be fuzzy, too), one may achieve a characteristic that is essential in modern engineering: robustness of the designed control system.

Finally, let

1) $\left[x_{0}, y_{0}\right]^{\mathrm{T}} \in \mathbb{R}^{2}$ and $\left[x_{T}, y_{T}\right]^{\mathrm{T}} \in \mathbb{R}^{2}$ represent initial and target states, respectively;

2) $\mathcal{W}$ denotes a fuzzy set with a support such that $\operatorname{supp}(\mathcal{W}) \subset[0,1)$

3) the difference equation

$$
\begin{aligned}
& \mathcal{X}_{j+1}=\mathcal{X}_{j}+\mathcal{Y}_{j} \\
& \mathcal{Y}_{j+1}=\mathcal{Y}_{j}-\mathcal{W} \operatorname{sgn}\left(\mathcal{Y}_{j}\right)+u_{j}
\end{aligned}
$$

and the initial condition

$$
\left[\begin{array}{l}
\mathcal{X}_{0} \\
\mathcal{Y}_{0}
\end{array}\right]=\left[\begin{array}{l}
x_{0} \\
y_{0}
\end{array}\right]
$$

describe the dynamics of a system with the fuzzy state $\left[\mathcal{X}_{j}, \mathcal{Y}_{j}\right]^{\mathrm{T}}$, submitted to the control $u_{j}$ limited to the interval $[-1,1]$.

The goal of this paper is to design a suboptimal (in respect to time) feedback controller, whose values directly depend only on the valid state of the object, obtained by a real-time measurement process.

The foregoing problem has been formulated in a fundamental version in order to assure the clarity of the investigations. Section $\mathrm{V}$ will provide example concepts of generalizations to forms frequently used in engineering applications. 


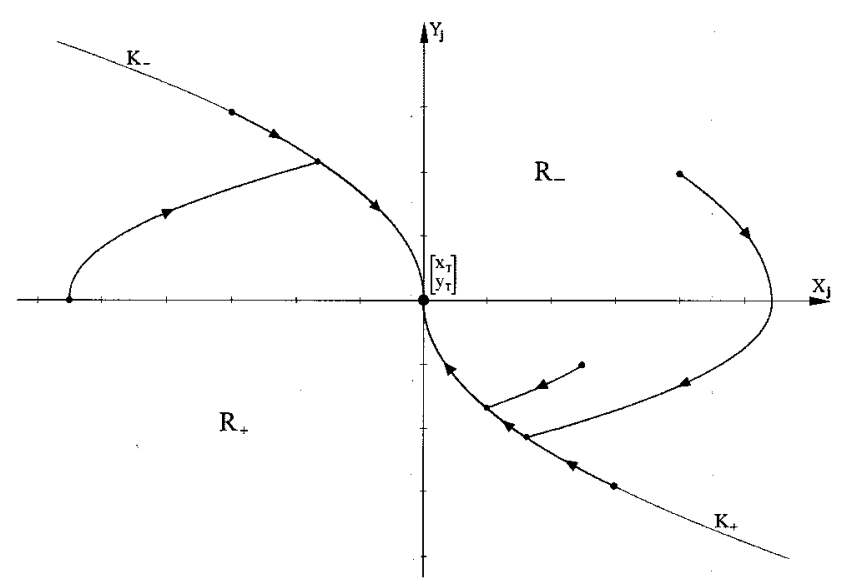

Fig. 1. Time-optimal controller (12) and trajectories in the case $y_{T}=0$.

\section{SOME AUXILIARY CONSIDERATIONS}

In the following section, an auxiliary task will be investigated. Let at this point the fuzzy set $\mathcal{W}$ be reduced to the real number $w$. Due to Assumption 2, $w \in[0,1)$.

Suppose that $\left[x_{+}, y_{+}\right]^{\mathrm{T}}$ and $\left[x_{-}, y_{-}\right]^{\mathrm{T}}$ are unique solutions [10] of the ordinary differential equation related then to system (3-4)

$$
\begin{aligned}
& \dot{x}(t)=y(t) \\
& \dot{y}(t)=u(t)-w \operatorname{sgn}(y(t))
\end{aligned}
$$

with the condition $\left[x_{+}(0), y_{+}(0)\right]^{\mathrm{T}}=\left[x_{-}(0), y_{-}(0)\right]^{\mathrm{T}}=$ $\left[x_{T}, y_{T}\right]^{\mathrm{T}}$, defined on the interval $(-\infty, 0]$, and generated by the control $u \equiv+1$ or $u \equiv-1$, respectively. Moreover, consider

$$
\begin{array}{ll}
K_{+}=\left\{\left[x_{+}(t), y_{+}(t)\right]^{\mathrm{T}},\right. & \text { for } t<0\} \\
K_{-}=\left\{\left[x_{-}(t), y_{-}(t)\right]^{\mathrm{T}},\right. & \text { for } t<0\}
\end{array}
$$

therefore, these are the sets of all states which can be brought to the target $\left[x_{T}, y_{T}\right]^{\mathrm{T}}$ by the control $u \equiv+1$ or $u \equiv-1$, respectively. Also let

$$
\begin{array}{r}
R_{+}=\left\{\left[\begin{array}{l}
x \\
y
\end{array}\right]\right. \text { such that there exists } \\
\left.\left[\begin{array}{c}
x^{*} \\
y
\end{array}\right] \in K \text { with } x>x^{*}\right\} \\
R_{-}=\left\{\begin{array}{l}
x \\
y
\end{array}\right] \text { such that there exists } \\
\\
\left.\left[\begin{array}{l}
x^{*} \\
y
\end{array}\right] \in K \text { with } x<x^{*}\right\}
\end{array}
$$

where $K=K_{-} \cup\left\{\left[x_{T}, y_{T}\right]^{\mathrm{T}}\right\} \cup K_{+}$. The time-optimal control is then expressed by the following [11]:

$$
\begin{aligned}
u(t) & =u_{r}(x(t), y(t)) \\
& = \begin{cases}-1, & \text { if }[x(t), y(t)]^{\mathrm{T}} \in\left(R_{-} \cup K_{-}\right) \\
0, & \text { if }[x(t), y(t)]^{\mathrm{T}} \in\left\{\left[x_{T}, y_{T}\right]^{\mathrm{T}}\right\} \\
+1, & \text { if }[x(t), y(t)]^{\mathrm{T}} \in\left(R_{+} \cup K_{+}\right)\end{cases}
\end{aligned}
$$

and the set $K$ constitutes a switching curve (Figs. 1 and 2).

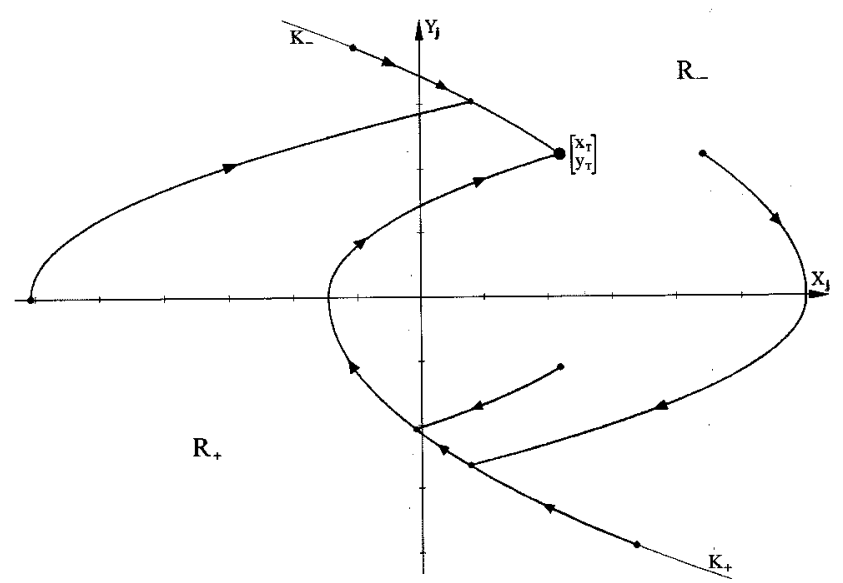

Fig. 2. Time-optimal controller (12) and trajectories in the case $y_{T} \neq 0$.

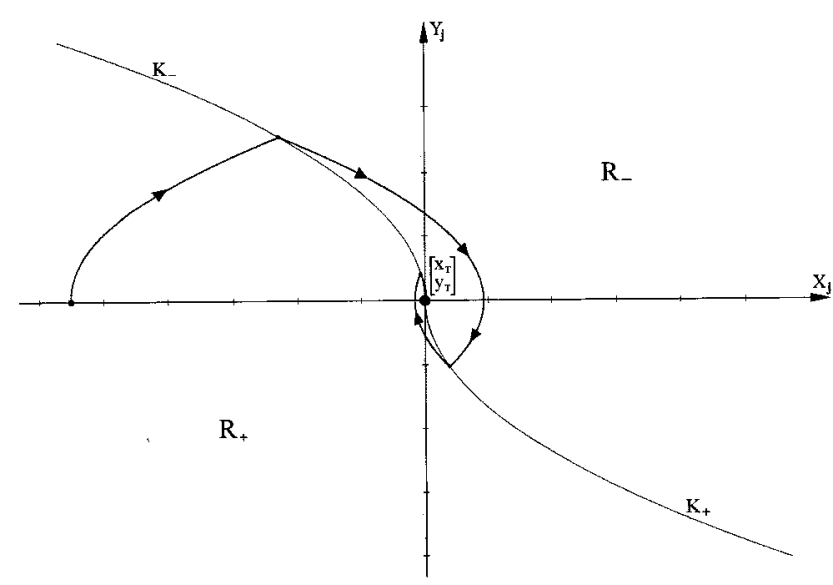

Fig. 3. Trajectories for $W>w$ in the case $y_{T}=0$.

In the time-optimal feedback controller equations, i.e., (8)-(12), the parameter $w$ intervenes, because it influences the form of the trajectories $\left[x_{+}, y_{+}\right]^{\mathrm{T}},\left[x_{-}, y_{-}\right]^{\mathrm{T}}$ and, therefore, also the shape of the switching curve $K$. In the fuzzy system, however, its value is of course not uniquely defined. The analysis of the system's sensitivity to the value of that parameter [11], which is briefly presented below, will then be of great importance. Thus, the value of the parameter $w$ occurring in the object is still denoted as $w$; however, the value used in feedback controller equations will be marked by $W$; therefore, the parameter $W$ can be interpreted as an (uncertain) knowledge about the parameter $w$ needed for the purpose of the synthesis of the feedback controller.

The case where the second coordinate of the target state is equal to zero, i.e., with $y_{T}=0$, will be considered first.

If $W=w$, the control is time-optimal (Fig. 1). The state of the system is brought to the switching curve, and being permanently included in this curve hereafter, it reaches the target in a minimal and finite time.

The trajectory representative for $W>w$ is shown in Fig. 3 . As a result of its having oscillations around the target, overregulations occur in the system. The target is reached in a finite time. 


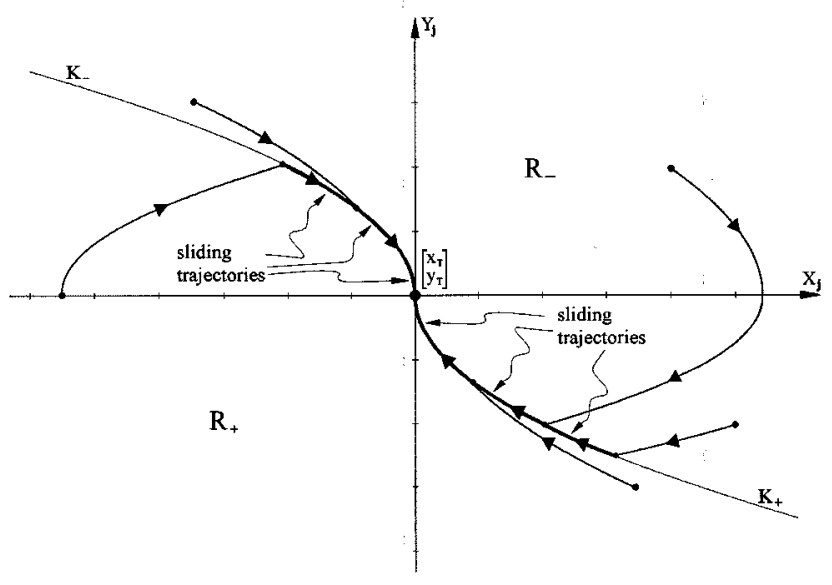

Fig. 4. Trajectories for $W<w$ in the case $y_{T}=0$.

Fig. 4 shows, however, the trajectories that are representative when $W<w$. After the switching curve is crossed, sliding trajectories [12] appear in the system. Here, too, the target is reached in a finite time.

In both of the last two cases, i.e., with $W \neq w$, the time to reach the target increases from the optimal more or less proportionally to the difference between the values $W$ and $w$.

The remaining case $y_{T} \neq 0$ will be presented now. Let, in particular, $y_{T}>0$; investigations for $y_{T}<0$ can be made analogously.

If $W=w$, the control is time-optimal (see again Fig. 2), and the considerations are identical as before for $y_{T}=0$.

When $W>w$, the trajectories occurring in the system create a limit cycle; the target is not reached (see Fig. 5).

Finally, for $W<w$ (Fig. 6) only some of the trajectories (marked on Fig. 6 with arrows) reach the target in a finite time. Other trajectories attain only the end point $\left[x_{E}, y_{E}\right]^{\mathrm{T}}$, which is the intersection of the axis $x$ and the switching curve; the state does not then reach the target. Sliding trajectories occur on the switching curve.

\section{Suboptimal FeEdBack CONTROLler FOR A FUZZY SYSTEM}

In this section, the fuzzy system (3)-(5), which is the subject of the present paper, will be considered. The parameter $w$, introduced in the previous section, happens to be a fuzzy set $\mathcal{W}$ in the problem worked out here. A fuzzy set naturally cannot be used directly to define a control in a real system. For this reason, some elements of fuzzy decision theory [13] will be used. Its aim is to make the optimal selection of one element from all possible decisions on the basis of a membership function.

Let the following be given: a fuzzy set $\mathcal{Z}$ (with the membership function $\mu_{Z}: \mathbb{R} \rightarrow[0, \infty)$ ) representing the state of reality, a nonempty set $D$ of possible decisions, and a loss function

$$
l: D \times \mathbb{R} \rightarrow \mathbb{R} \cup\{ \pm \infty\}
$$

where the values $l(d, z)$ can be interpreted as losses occurring in the hypothetical case when the fuzzy set $\mathcal{Z}$ is reduced to the

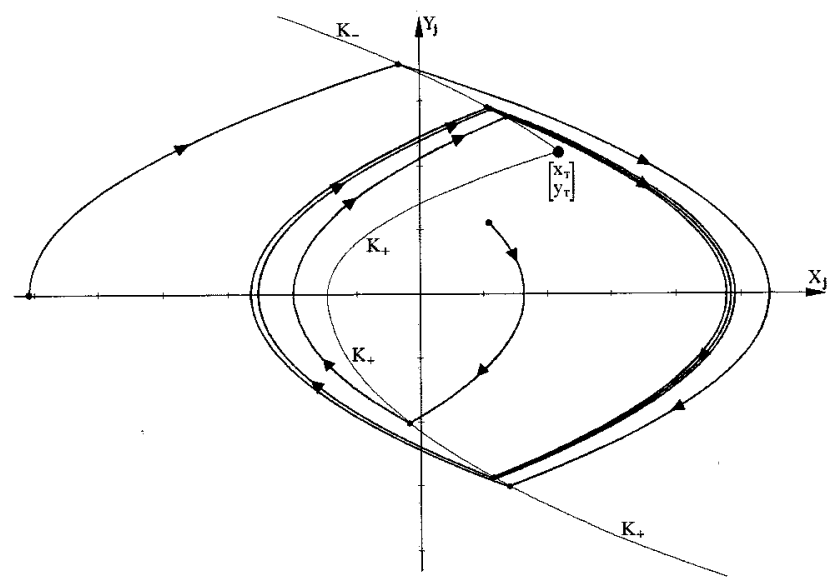

Fig. 5. Trajectories for $W>w$ in the case $y_{T} \neq 0$.

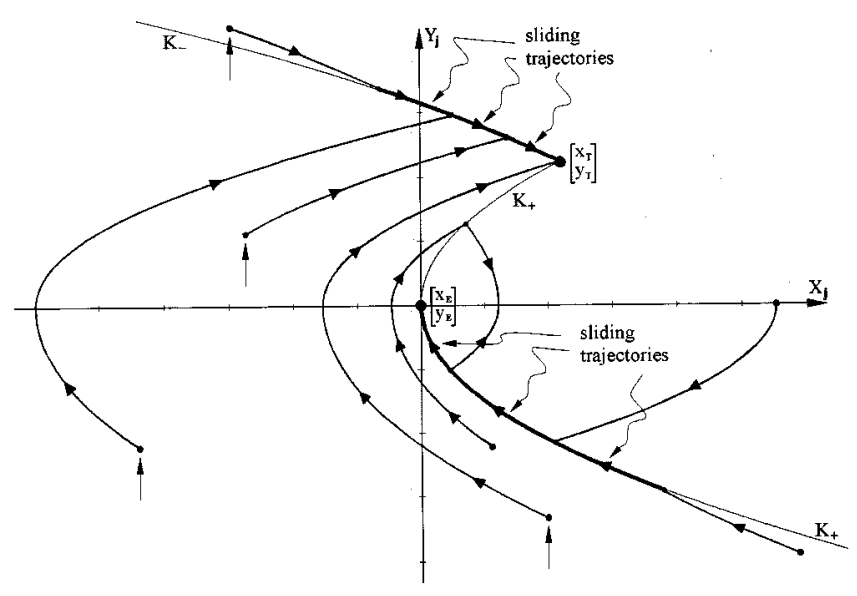

Fig. 6. Trajectories for $W<w$ in the case $y_{T} \neq 0$.

real number $z$, and the decision $d$ has been made. Denote by $l_{m}: D \rightarrow \mathbb{R} \cup\{ \pm \infty\}$ the minimax loss function

$$
l_{m}(d)=\sup _{z \in \operatorname{supp}(z)} l(d, z) .
$$

If additionally for every $d \in D$ the integral $\int_{\mathbf{R}} l(d, z) \mu_{\mathcal{Z}}(z) d z$ exists, suppose also the Bayes loss function $l_{b}: D \rightarrow \mathbb{R} \cup\{ \pm \infty\}$ defined as

$$
l_{b}(d)=\int_{\mathbf{R}} l(d, z) \mu_{\mathcal{Z}}(z) d z .
$$

Every element $d_{m} \in D$ such that

$$
l_{m}\left(d_{m}\right)=\inf _{d \subset D} l_{m}(d)
$$

is called a minimax decision and, analogously, every element $d_{b} \in D$ such that

$$
l_{b}\left(d_{b}\right)=\inf _{d \in D} l_{b}(d)
$$

is called a Bayes decision. The procedures for obtaining these elements are said to be minimax and Bayes rules, respectively. The main difference between the above rules appears in their 
interpretation. This results directly from the forms of the functions $l_{m}$ and $l_{b}$ : the "pessimistic" minimax rule assumes the occurrence of the most unfavorable state of reality and counteracts it, while the Bayes rule is more flexible.

In the problem of a time-optimal control investigated here, the parameter $W \in D \subset[0,1)$ used in the feedback controller equations will be treated as a decision, and the number $\mathcal{W}$ occurring in system (3-4), as the fuzzy state of reality. The loss function (13) is defined for $(W, w) \in D \times \mathbb{R}$, and its values are related to the time to reach the target, if in the feedback controller equations the parameter $W$ was used, while hypothetically in the object the value $w$ is occurring.

Once again the case $y_{T}=0$ will be considered first. The following suggestions for the determination of the value of the parameter $W$ result from the analysis of the auxiliary problem presented in the previous section.

If overregulations can be allowed, it is worthwhile using the Bayes rule with real values for the loss function. Such a choice is possible because the determination of the parameter $W$ value that is either less than, equal to, or greater than $w$ allows the system state to be brought to the target in a finite time. (However, this time increases approximately proportionally to the difference between the values $W$ and $w$.)

If overregulations are not allowed, this determination should be carried out on the basis of the minimax rule, assuming infinite values of the loss function for $W>w$. This enables the overregulations to be avoided, because they occur only if $W>w$.

Let now $y_{T} \neq 0$. The case $y_{T}>0$ will be considered; the investigations for $y_{T}<0$ are analogous.

The condition $W=w$ is impossible to obtain in practice. However, the determination of a parameter $W$ value that is either greater or less than $w$ precludes reaching the target from any initial state, because of the occurrence of the cycle (Fig. 5) or the existence of the end point (Fig. 6). In the proposed feedback controller, the switching curve $K$ will be divided into three parts. The division points are the target and the point of intersection with the axis $x$. For every part, there will be differently determined values of the parameter $W$, which for particular parts are defined in the following as $W_{1}, W_{2}$, and $W_{3}$.

The value of the parameter $W_{1}$, i.e., the one that defined the part of the switching curve $K_{-}$, which means $K$ for $y \in$ $\left[y_{T}, \infty\right)$ (see also Fig. 9), should be determined using the minimax rule with infinite values of the loss function for $W>w$. This choice is made in order to avoid the generation of a limit cycle, which appears when the value of the parameter $W_{1}$ is greater than $w$. If, however, this value is smaller than $w$, the state of the system is brought to the target in a finite time.

For the determination of the value of the parameter $W_{2}$ defining the part $K_{+}$for $y \in\left[0, y_{T}\right]$, it is necessary to apply the minimax rule with infinite values of the loss function for $W<w$. This is because an overly large value of the parameter $W_{2}$ allows the state to be brought to the part defined by the parameter $W_{1}$, which, as was demonstrated above, can be successfully determined. An overly small one, however, causes the occurrence of the end point, whose existence is not admissible from the point of view of utility.

Finally, the value of the parameter $W_{3}$ defining the part $K_{+}$ for $y \in(-\infty, 0)$ can be obtained using the Bayes rule with real

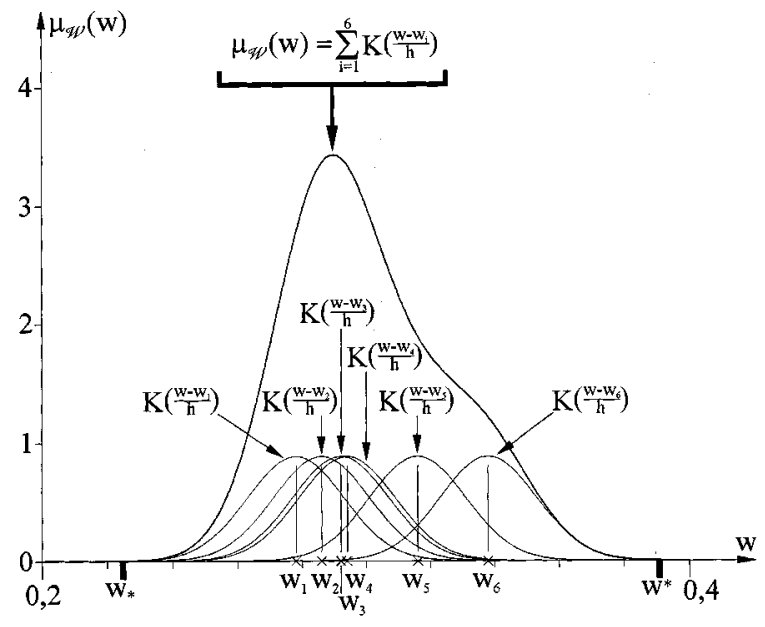

Fig. 7. Membership function $\mu_{W}$, specified using the kernel estimators technique [14], for experimentally obtained values of resistance to motion $w_{1}, w_{2}, \cdots, w_{6} ;(K-$ Gauss curve with restricted "tails," $h=0.08)$.

values of the loss function. Both overly small and overly large values of this parameter are acceptable, because this allows the state to be brought to the parts defined by the parameters $W_{1}$ and $W_{2}$, which can be successfully determined as shown above.

Suppose, as an example, that the fuzzy set $\mathcal{W}$ has the support of the form $\operatorname{supp}(\mathcal{W})=\left[w_{*}, w^{*}\right] \subset[0,1)$, and moreover let its membership function $\mu_{\mathcal{W}}$ be continuous and positive in the interval $\left(w_{*}, w^{*}\right)$. (The exemplary membership function, specified using the kernel estimators technique [14], for experimentally obtained values of resistance to motion is shown in Fig. 7; for the sake of clarity, the basic form with only six independent measurements has been used.) The loss function (13) will be described by the following:

$$
l(W, w)= \begin{cases}-p(W-w) & \text { if } W-w \leq 0 \\ q(W-w) & \text { if } W-w \geq 0\end{cases}
$$

where $p, q \in \mathbb{R}_{+} \cup\{\infty\}$, but only one of them can be infinite; in this case, let $\infty \cdot 0=0$.

According to the above assumptions, it is accepted that $D=$ $\left[w_{*}, w^{*}\right]$.

With the fixed value of the parameter $W$, from the definitions of minimax and Bayes loss functions (14) and (15), the following results, respectively:

$$
\begin{aligned}
l_{m}(W)= & \max \left(\left\{-p\left(W-w^{*}\right), q\left(W-w_{*}\right)\right\}\right) \\
l_{b}(W)= & \int_{w_{*}}^{W} q(W-w) \mu_{\mathcal{W}}(w) d w \\
& -\int_{W}^{w^{*}} p(W-w) \mu_{\mathcal{W}}(w) d w .
\end{aligned}
$$

If $p=\infty$, then from (19) it can be determined that the infimum of the function $l_{m}$ on the set $D$ is realized by

$$
W=w^{*}
$$

and if $q=\infty$, then this infimum is assumed for

$$
W=w_{*} \text {. }
$$


The values $W$ indicated by (21) and (22) constitute the desired minimax decision with infinite values of the loss function for $W<w$ and $W>w$, respectively.

With real positive values $p$ and $q$, however, the function $l_{b}$ is differentiable in the interval $\left(w_{*}, w^{*}\right)$; therefore, one obtains

$$
l_{b}^{\prime}(W)=p \int_{w^{*}}^{W} \mu_{\mathcal{W}}(w) d w+q \int_{w_{*}}^{W} \mu_{\mathcal{W}}(w) d w
$$

as well as

$$
l_{b}^{\prime \prime}(W)=(p+q) \mu_{\mathcal{W}}(w)
$$

Using (23), the equivalence of the following conditions can be proved by elementary transformations:

$$
\begin{aligned}
l_{b}^{\prime}(W) & =0 \\
\int_{w_{*}}^{W} \mu_{\mathcal{W}}(w) d w & =\frac{p}{p+q} \int_{w_{*}}^{w^{*}} \mu_{\mathcal{W}}(w) d w .
\end{aligned}
$$

Equation (24) implies that the function $l_{b}^{\prime \prime}$ is positive in the set $\left(w_{*}, w^{*}\right)$; therefore, the function $l_{b}$ is here strictly convex. Because $0<(p / p+q)<1$, (26), equivalent to condition (25), is fulfilled only at one point; at this point, then, the function $l_{b}$ assumes its minimum, global in the set $D=\left[w_{*}, w^{*}\right]$ due to the continuity of this function at the points $w_{*}$ and $w^{*}$. Finally, the value $W$ that fulfills condition (26) constitutes the desired Bayes decision with real values of the loss function. To obtain this value one can use the kernel estimators technique, according to the algorithm presented in papers [15], [16]. Finally, the proof of condition (26), omitting the assumption of the continuity of the membership function $\mu \mathcal{W}$, is found in article [16].

To summarize, in accordance with the considerations stated before, if the values of the parameters $W$ or $W_{1}, W_{2}, W_{3}$ should be determined due to the minimax rule with infinite values of the loss function for $W<w$ or $W>w$ or the Bayes rule with real values of this function, then they can be obtained from (21), (22), or (26), respectively.

If one possesses the obtained values $W$ or $W_{1}, W_{2}, W_{3}$, the feedback controller equations can be calculated. Thus, the equations of the switching curve $K$ take on the form

$$
\begin{aligned}
& x=\frac{y^{2}-y_{T}^{2}}{2\left(-1-W_{1}\right)}+x_{T} \quad \text { for } y \in\left[y_{T}, \infty\right) \\
& x=\frac{y^{2}-y_{T}^{2}}{2\left(1-W_{2}\right)}+x_{T} \quad \text { for } y \in\left[0, y_{T}\right] \\
& x=\frac{y^{2}}{2\left(1-W_{3}\right)}-\frac{y_{T}^{2}}{2\left(1-W_{2}\right)}+x_{T} \quad \text { for } y \in(-\infty, 0]
\end{aligned}
$$

in the case $y_{T}>0$. [(27) defines the set $K_{-}$, while dependencies (28) and (29), the set $K_{+}$.] For $y_{T}<0$, the equations are analogous. If $y_{T}=0$, one should substitute, in (27) and (29), $W=W_{1}=W_{3}$ (dependence (28) has no meaning here). The sets $R_{-}$and $R_{+}$constitute adequate areas resulting from the section of the plane $\mathbb{R}^{2}$ by the curve $K$, according to (10) and

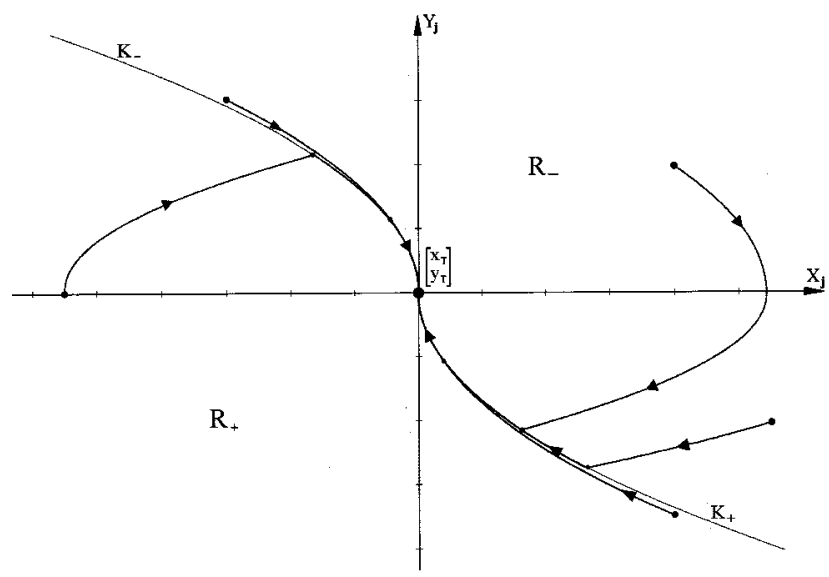

Fig. 8. Fuzzy feedback controller (30) and trajectories in the case $y_{T}=0$.

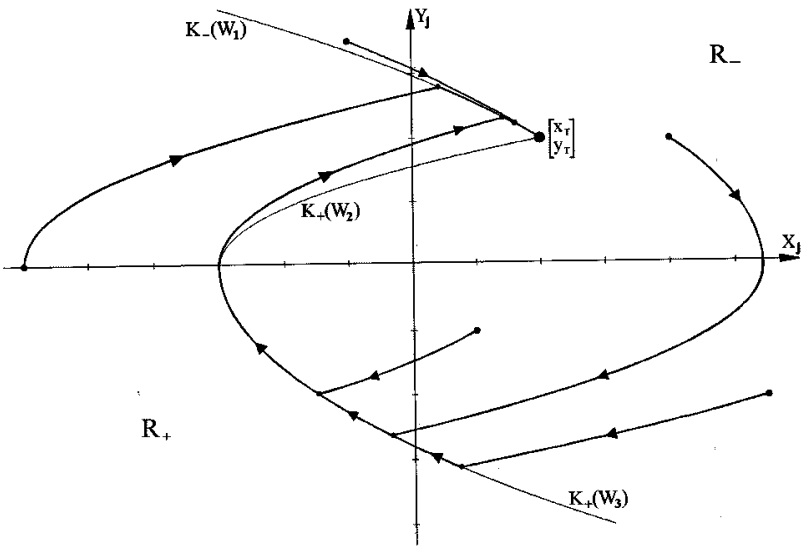

Fig. 9. Fuzzy feedback controller (30) and trajectories in the case $y_{T} \neq 0$.

(11). For the sets $K_{-}, K_{+}, R_{-}, R_{+}$obtained in this way, the value of the control is simply defined by

$$
u_{j}= \begin{cases}-1, & \text { if }\left[x_{j}, y_{j}\right]^{\mathrm{T}} \in\left(R_{-} \cup K_{-}\right) \\ 0, & \text { if }\left[x_{j}, y_{j}\right]^{\mathrm{T}} \in\left\{\left[x_{T}, y_{T}\right]^{\mathrm{T}}\right\} \\ +1, & \text { if }\left[x_{j}, y_{j}\right]^{\mathrm{T}} \in\left(R_{+} \cup K_{+}\right)\end{cases}
$$

where $\left[x_{j}, y_{j}\right]^{\mathrm{T}}$ means the object state, obtained by a real-time measurement process in the moment $j$. Figs. 8 and 9 provide an illustration of the control structure worked out here with the trajectories it generates.

The control designed above may lead to chattering, i.e., frequent switchings between the two values +1 and -1 along sliding trajectories. In mechanical systems, such a phenomena can have a negative impact on the actuator life and excite vibrations in elastic transmissions, hence, it should be avoided. Under the assumption that the control may take any value in the interval $[-1,1]$, this goal can be obtained by substituting a modified control law, rendered continuous instead of discontinuous (30).

As before, the case $y_{T}=0$ will be considered first. Initially, the next parameter $W^{\sim}$ can be introduced, in addition to the constant $W$ used so far, with the condition $-1<W^{\sim}<W$. Let also, beside the sets $K_{-}$and $K_{+}$defined by (8) and (9) for the parameter $w$ (or more precisely $W$ ), similar sets $K_{-}^{\sim}$ 


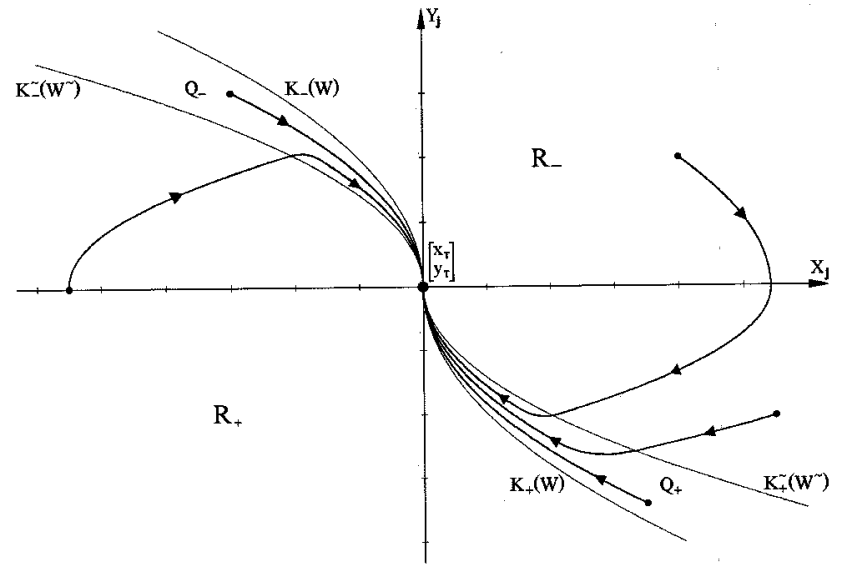

Fig. 10. Fuzzy feedback controller (35) and trajectories in the case $y_{T}=0$.

and $K_{+}^{\sim}$ be given for the constant $W$ (see Fig. 10). Defining moreover

$$
\begin{aligned}
Q_{-}= & \left\{\left[\begin{array}{l}
x \\
y
\end{array}\right] \text { such that there exists }\left[\begin{array}{c}
x^{*} \\
y
\end{array}\right] \in K_{-}^{\sim}\right. \text { and } \\
& {\left.\left[\begin{array}{c}
x^{* *} \\
y
\end{array}\right] \in K_{-} \text {with } x^{*} \leq x \leq x^{* *}\right\} } \\
Q_{+}= & \left\{\left[\begin{array}{l}
x \\
y
\end{array}\right] \text { such that there exists }\left[\begin{array}{c}
x^{*} \\
y
\end{array}\right] \in K_{+}\right. \text {and } \\
& {\left.\left[\begin{array}{c}
x^{* *} \\
y
\end{array}\right] \in K_{+}^{\sim} \text { with } x^{*} \leq x \leq x^{* *}\right\} }
\end{aligned}
$$

where $Q=Q_{-} \cup\left\{\left[x_{T}, y_{T}\right]^{\mathrm{T}}\right\} \cup Q_{+}$, and slightly altering (10) and (11) to

$$
\begin{aligned}
R_{+}= & \left\{\left[\begin{array}{l}
x \\
y
\end{array}\right] \in \mathbb{R}^{2} \backslash Q\right. \text { such that there exists } \\
& {\left.\left[\begin{array}{c}
x^{*} \\
y
\end{array}\right] \in Q \text { with } x<x^{*}\right\} } \\
R_{-}= & \left\{\left[\begin{array}{l}
x \\
y
\end{array}\right] \in \mathbb{R}^{2} \backslash Q\right. \text { such that there exists } \\
& {\left.\left[\begin{array}{c}
x^{*} \\
y
\end{array}\right] \in Q \text { with } x>x^{*}\right\} }
\end{aligned}
$$

the time-optimal control can now be given by

$$
u_{j}= \begin{cases}-1, & \text { if }\left[x_{j}, y_{j}\right]^{\mathrm{T}} \in R_{-} \\ -z\left(-x_{j},-y_{j}\right), & \text { if }\left[x_{j}, y_{j}\right]^{\mathrm{T}} \in Q_{-} \\ 0, & \text { if }\left[x_{j}, y_{j}\right]^{\mathrm{T}} \in\left\{\left[x_{T}, y_{T}\right]^{\mathrm{T}}\right\} \\ z\left(x_{j}, y_{j}\right), & \text { if }\left[x_{j}, y_{j}\right]^{\mathrm{T}} \in Q_{+} \\ +1, & \text { if }\left[x_{j}, y_{j}\right]^{\mathrm{T}} \in R_{+}\end{cases}
$$

with the function $z: \mathbb{R}^{2} \rightarrow \mathbb{R}$ continuously and strictly increasing from the value -1 on the sets $K_{-}^{\sim}$ and $K_{+}$to the value 1 on the sets $K_{-}$and $K_{+}^{\sim}$. A suitable value for the parameter $W^{\sim}$ can be determined heuristically; in general, the difference $W-W^{\sim}$ should be proportional to the delay in the system. The trajectories generated by control (35) are shown in Fig. 10 for $y_{T}=0$. Thus, control law (35) constitutes the continuated variant of control (30), which is of the "bang-bang" type.

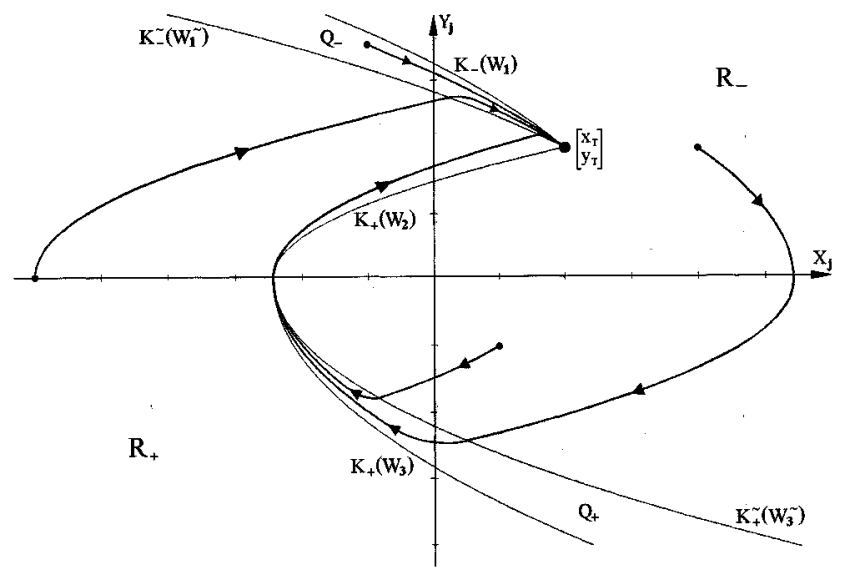

Fig. 11. Fuzzy feedback controller (35) and trajectories in the case $y_{T} \neq 0$.

And now, the second case $y_{T} \neq 0$ will be worked out. As previously, in addition to the parameters $W_{1}, W_{3}$ defined earlier, two further constants $W_{1}^{\sim}, W_{3}^{\sim}$ must be determined heuristically according to the delay in the system, subject to the conditions $-1<W_{1}^{\sim}<W_{1},-1<W_{3}^{\sim}<W_{3}$. The concept introduced in the preceding paragraph, expressed by (35), should be applied here twice in a natural way. An illustration of the control structure such obtained, along with the trajectories it generates, is provided in Fig. 11.

\section{Simulation Results}

The correct functioning of the suboptimal structures described in this paper has been verified by numerical simulations. The object was a single-degree-of-freedom mechanical system (1) with the discontinuous model of resistance to motion given in the form of the so-called Stribeck curve [19], obtained experimentally from a metallurgic reversing mill. Random components with expectation values $2-10 \%$ of proper averages were added to the particular quantities, representing perturbations and noise occurring in the system.

Typical results obtained for control structures (30) and (35) worked out in this paper are shown in Figs. 8-11. If it is assumed that over-regulations are undesirable, then they did not occur in the controlled object, though the times to reach the target set were then greater than those obtained without this limit. In conclusion, it should be strongly emphasized that the control structures presented in this paper turn out to be only slightly sensitive to the inaccuracy resulting from identification and the occurrence of perturbations. Such robustness should be emphasized as a very valuable property of uncertain, especially fuzzy, control systems.

Table I contains a comparison of the times obtained using feedback controllers (30), (35) designed in this paper, classical sliding mode control [18], and a friction compensation system (similar to that presented in [19] for an inverted pendulum). The shortest times were obtained using structure (30). The results obtained by continuated structure (35) were comparable, though 1-3\% worse. Finally, the times to reach the target set for the sliding mode control were 5-10\% greater, which seems obvious due to the lack of optimal selection of parameter values. 
TABLE I

Times to Reach the Target Set, Obtained Using Feedback Controllers (30), (35), Sliding Mode Control, and Friction Compensation System

\begin{tabular}{|c|c|c|c|c|c|}
\hline \multicolumn{2}{|c|}{ Initial and target states } & \multirow{2}{*}{$\begin{array}{c}\text { Feedback } \\
\text { controller } \\
(30)\end{array}$} & \multirow{2}{*}{$\begin{array}{c}\text { Feedback } \\
\text { controller } \\
(35)\end{array}$} & \multirow{2}{*}{$\begin{array}{l}\text { Sliding } \\
\text { mode } \\
\text { control }\end{array}$} & \multirow{2}{*}{$\begin{array}{l}\text { Friction } \\
\text { compen- } \\
\text { sation }\end{array}$} \\
\hline$\left[x_{0}, y_{0}\right]^{\mathrm{T}}$ & {$\left[x_{T}, y_{T}\right]^{\mathrm{T}}$} & & & & \\
\hline$[2,0]^{\mathrm{T}}$ & {$[0,0]^{\mathrm{T}}$} & $2.89(0 \%)$ & $2.93(+1.4 \%)$ & $3.04(+5.1 \%)$ & $3.24(+12.1 \%)$ \\
\hline$[5,0]^{\mathrm{T}}$ & {$[0,0]^{\mathrm{T}}$} & $4.58(0 \%)$ & $4.67(+1.9 \%)$ & $4.99(+9.0 \%)$ & $5.40(+17.9 \%)$ \\
\hline$[10,0]^{\mathrm{T}}$ & {$[0,0]^{\mathrm{T}}$} & $6.51(0 \%)$ & $6.68(+2.6 \%)$ & $7.18(+10.3 \%)$ & $7.48(+14.9 \%)$ \\
\hline$[-5,0]^{\mathrm{T}}$ & {$[2,2]^{\mathrm{T}}$} & $4.38(0 \%)$ & $4.45(+1.6 \%)$ & $4.67(+6.6 \%)$ & $4.84(+10.5 \%$ \\
\hline$[-5,-2]^{\mathrm{T}}$ & {$[2,2]^{\mathrm{T}}$} & $6.58(0 \%)$ & $6.77(+2.9 \%)$ & $7.22(+9.7 \%)$ & $7.71(+17.2 \%)$ \\
\hline
\end{tabular}

The least satisfactory results were obtained for the friction compensation system, which can be explained by the general difficulties entailed in using such methods for tasks with bounded control (since, generally speaking, it is necessary here to apply a yet sharper boundary in order to assure a certain reserve that will allow for possible compensation of friction; once obtained practically in this manner, the control values can then be far from maximal).

\section{SUMMARY AND FINAL REMARKS}

This paper has dealt with the problem of time-optimal control for single-degree-of-freedom mechanical systems with the discontinuous and uncertain model of resistance to motion. A fuzzy approach has been used in the design of discrete-time control laws (30) and (35), in discontinuous and continuous versions, respectively.

As mentioned in Section I, the problem worked out here has been formulated in a fundamental version in order to assure the clarity of the investigations. However, the material presented here allows for the easy introduction of generalizations to forms that are frequently used in engineering applications.

First, let $u$, introduced in (1), mean the momentum obtained from the drive, which is treated here as a inertial element with the constant $T$, i.e.,

$$
\dot{u}(T)=-\frac{1}{T} u(t)+v(t)
$$

and subsequently let $v$ be a bounded control (for the engineering basics and interpretation, see, e.g., [1, ch. 7.4] and [20]). If the number $T$ is treated as the fuzzy set $\mathcal{T}$, the concept of the feedback controller presented here can easily be generalized to a system constructed in this way. An analysis of sensitivity to the value of the parameter $T$ produces results similar to those presented in Section II: namely, an overly large value used for the feedback controller equations implies sliding trajectories, while too small value generates limit cycles. Of course, due to the increase in the dimension of the vector state to three, the switching curve crosses into the switching surface and the analysis becomes much more complex, but the basic principles remained unchanged in terms of the fundamental concept presented earlier in this paper.

Once again, let the basis for considerations constitute the fundamental system described in Section I. If one transforms (1) to the form

$$
J \ddot{x}(t)+B \dot{x}(t)=h(\cdot)+u(t)
$$

then one obtains the well-known model of a DC motor with the friction model $h$ and the constants $B$ describing viscous damping as well as $J$ representing the momentum of inertia (for the engineering basics and interpretation, see, e.g., [2, sec. 36] and [21]). Here, too, by treating the parameters $B$ and $J$ as the fuzzy sets $\mathcal{B}$ and $\mathcal{J}$, the concept presented in this paper can be generalized for purposes of synthesizing the time-optimal control, in accordance with the scheme presented in the previous section. Here, also, the occurrence of sliding trajectories and limit cycles is dependent on the value of those parameters used for feedback controller equations. The differences are rather of a technical nature, e.g., the shapes of trajectories are variable, which naturally implies a different type of formulas (27)-(29).

Finally, the concept presented here can be used in a similar fashion for many other models of mechanical systems frequently encountered in engineering applications.

\section{REFERENCES}

[1] M. Athans and P. L. Falb, Optimal Control. New York: McGraw-Hill, 1966.

[2] W. G. Boltianski, Mathematical Methods of Optimal Control. Moscow, USSR: Nauka, 1969.

[3] A. Weinmann, Uncertain Models and Robust Control. Berlin, Germany: Springer-Verlag, 1991 
[4] K. Zhou, J. C. Doyle, and K. Glover, Robust and Optimal Control. Englewood Cliffs, NJ: Prentice-Hall, 1996.

[5] R. Gorez and M. De Neyer, "Fuzzy control of robotic manipulators and mechanical systems," in Fuzzy Reasoning in Information, Decision and Control Systems, S. G. Tzafestas and A. N. Venetsnopoulos, Eds. Dordrecht, The Netherlands: Kluwer, 1994, pp. 451-492.

[6] P. Kulczycki, "Almost certain time-optimal positional control," IMA J. Mathematical Contr. Inform., vol. 13, pp. 63-77, 1996.

[7] — , "A random approach to time-optimal control," J. Dynamic Syst., Meas., Contr., vol. 121, pp. 542-543, 1999.

[8] B. Armstrong, "Challenges to systematically engineered friction compensation," in Proc. IFAC Workshop Motion Contr., Munich, Germany, Oct. 1995, pp. 21-30.

[9] - Control of Machines with Friction. Dordrecht, The Netherlands: Kluwer, 1991.

[10] P. Kulczycki, "Some remarks on solutions of discontinuous differential equations applied in automatic control," Indust. Math., vol. 46, pp. 119-128, 1996.

[11] — - Time-Optimal Stochastic Control of Discontinuous Dynamical Systems. Cracow, Poland: WPK, 1992.

[12] J.-J. E. Slotine and W. Li, Applied Nonlinear Control. Englewood Cliffs, NJ: Prentice-Hall, 1991.

[13] J. Kacprzyk, Fuzzy Sets in System Analysis. Warsaw, Poland: PWN, 1986.
[14] B. L. S. Prakasa Rao, Nonparametric Functional Estimation. New York: Academic, 1983

[15] H. Schiøler and P. Kulczycki, "Neural network for estimating conditional distributions," IEEE Trans. Neural Networks, vol. 8, pp. 1015-1025, Sept. 1997.

[16] P. Kulczycki, "Parameter identification using Bayes and kernel approaches," Proc. Nat. Sci. Council ROC(A), vol. 23, pp. 205-213, 1999.

[17] J. T. Teeter, M.-Y. Chow, and J. J. Brickley, Jr., "A novel fuzzy friction compensation approach to improve the performance of a DC motor control system," IEEE Trans. Indust. Electron., vol. 43, pp. 113-120, Feb. 1996.

[18] V. I. Utkin, Sliding Models in Control Optimization. Berlin, Germany: Springer-Verlag, 1992.

[19] E. Ostertag and M. J. Carvalho-Ostertag, "Fuzzy control of an inverted pendulum with fuzzy compensation of friction forces," Int. J. Syst. Sci., vol. 24, pp. 1915-1921, 1993.

[20] W. Hejmo and P. Kulczycki, "On the approach to time-optimal control of positional systems," Archiwum Automatyki i Telemechaniki, vol. XXXI, pp. 103-116, 1986.

[21] L. Güvenç, "Friction compensation using fuzzy logic control," in Proc. Int. Conf. ICRAM'95 Recent Adv. Mechatron., vol. 2, Istanbul, Turkey, Aug. 1995, pp. 667-672. 